\title{
MAGNETIC FLUX-CURRENT SURFACES OF MAGNETOHYDROSTATIC EQUILIBRIA
}

\author{
G. S. ChOE $^{1,2}$ And Minhwan JanG ${ }^{2,1}$ \\ ${ }^{1}$ School of Space Research, Kyung Hee University, Yongin 446-701, Korea \\ E-mail : gchoe@khu.ac.kr \\ 2 Department of Astronomy and Space Science, Kyung Hee University, Yongin 446-701, Korea \\ (Received December 2, 2013; Accepted December 8, 2013)
}

\begin{abstract}
Magnetohydrostatic equilibria, in which the Lorentz force, the plasma pressure force and the gravitational force balance out to zero, are widely adopted as the zeroth order states of many astrophysical plasma structures. A magnetic flux-current surface is a surface, in which both magnetic field lines and current lines lie. We for the first time derive the necessary and sufficient condition for existence of magnetic flux-current surfaces in magnetohydrostatic equilibria. It is also shown that the existence of flux-current surfaces is a necessary (but not sufficient) condition for the ratio of gravity-aligned components of current density and magnetic field to be constant along each field line. However, its necessary and sufficient condition is found to be very restrictive. This finding gives a significant constraint in modeling solar coronal magnetic fields as force-free fields using photospheric magnetic field observations.
\end{abstract}

Key words : magnetohydrodynamics (MHD) — methods: analytical — Stars: magnetic fields — Sun: atmosphere

\section{INTRODUCTION}

Plasmas in most parts of the universe are magnetized and under the influence of gravity. The zeroth order state of such an astrophysical plasma structure would be a magnetohydrostatic (hereafter MHS) equilibrium, in which the Lorentz force, the plasma pressure gradient force and the gravitational force balance out to zero (see e.g., Parker 1979). The equations governing the MHS equilibrium are

$$
\begin{gathered}
\boldsymbol{J} \times \boldsymbol{B}-\nabla p-\rho \nabla \Phi=0, \\
\boldsymbol{J}=\nabla \times \boldsymbol{B}, \\
\nabla \cdot \boldsymbol{B}=0,
\end{gathered}
$$

where $\boldsymbol{B}$ is the magnetic field, $\boldsymbol{J}$ the electric current density, $p$ the plasma pressure, $\rho$ the plasma mass density, and $\Phi$ the gravitational potential. In Eq. 2, which is Ampère's law, we have omitted a factor $c /(4 \pi)$ (in Gaussian units) or $1 / \mu_{0}$ (in SI units) in front of $\nabla \times \boldsymbol{B}$ by an appropriate normalization (see e.g., Choe $\&$ Cheng 2000). The gravitational field or gravitational acceleration is given by

$$
\boldsymbol{g}=-\nabla \Phi .
$$

To solve the MHS equilibrium problem, we need to connect $p$ and $\rho$ through the equation of state

$$
p=R \rho T,
$$

Corresponding Author: G. S. Choe where $R$ is the gas constant. The equation of state introduces another variable, the temperature $T$, into the system. The temperature is governed by thermodynamics and is usually externally imposed for the closure of the system.

When the gravity is ignored, the MHS equation takes a very simple form:

$$
\boldsymbol{J} \times \boldsymbol{B}=\nabla p
$$

Applying a dot product with $\boldsymbol{B}$ and $\boldsymbol{J}$, respectively, to Eq. 6, we have

$$
\begin{aligned}
& \boldsymbol{B} \cdot \nabla p=0, \\
& \boldsymbol{J} \cdot \nabla p=0 .
\end{aligned}
$$

These equations imply that $p$ is constant along magnetic field lines and along current lines; in other words, the magnetic field lines and the current lines lie on constant $p$ surfaces. Such surfaces are called "magnetic flux surfaces" (Grad \& Rubin 1958). In an MHS equilibrium without gravity, the plasma pressure $p$ can thus be a flux surface label. Usually, the term "magnetic flux surface" has a broader meaning than the above. It is defined by D'haeseleer et al. (1991) as a surface that is traced out by a series of magnetic field lines. To avoid ambiguity, we will use the term "magnetic flux-current surface" to denote a surface, in which both magnetic field lines and current lines lie. The term "magnetic flux surface" will be used to designate a surface, in which magnetic field lines lie, and the term "current surface" to designate a surface, in which current lines 
lie. Therefore, a magnetic flux-current surface exists only when a magnetic flux surface and a current surface overlap. We will from time to time omit the word "magnetic" in front of "flux" as is often done in literatures.

When both the plasma pressure force and the gravitational force are ignorable compared to the Lorentz force, Eq. 1 becomes

$$
\boldsymbol{J} \times \boldsymbol{B}=0
$$

which represents a (Lorentz) force-free state. This equation implies that

$$
\boldsymbol{J}=\alpha \boldsymbol{B},
$$

where $\alpha$ is a certain scalar field, and it can be easily shown that

$$
\boldsymbol{B} \cdot \nabla \alpha=\boldsymbol{J} \cdot \nabla \alpha=0 .
$$

In a force-free field, the magnetic flux surface and the current surface always overlap to form a flux-current surface.

The coronal magnetic fields in the sun are often approximated as force-free fields (for the most recent reviews see Wiegelmann \& Sakurai 2012; Wheatland \& Gilchrist 2013; see also Aly 1989; Sakurai 1989; Amari et al. 1997; McClymont et al. 1997; Schrijver et al. 2006) because the plasma beta, the ratio of plasma pressure and magnetic pressure, is considered to be generally much smaller than unity in the solar corona. This force-free modeling of coronal magnetic fields generally adopts $B_{n}=\boldsymbol{B} \cdot \hat{\boldsymbol{n}}$, where $\hat{\boldsymbol{n}}$ is a boundary normal unit vector, and $\alpha=J_{n} / B_{n}$, where $J_{n}=\boldsymbol{J} \cdot \hat{\boldsymbol{n}}$, both observed in the photosphere as boundary conditions. Between the photosphere and the coronal base, there is a region, where the force-free assumption is invalid. Therefore, using $B_{n}$ and $\alpha$ observed in the photosphere as boundary conditions of the corona may generate serious concerns about the validity of force-free modeling of coronal fields.

In this paper, we emphasize that the existence of magnetic flux-current surfaces is a decisive factor in assessing the validity of coronal force-free modeling with photospheric data as boundary conditions. If magnetic flux-current surfaces exist in the region between the photosphere and the coronal base, a flux tube surrounded by a flux-current surface will have a constant magnetic flux and a constant current along the tube axis. Although the value of $\alpha$ may not be conserved field line by field line, the average value of $\alpha$ over a cross-section of the flux tube is conserved from the photosphere to the corona. This can more or less justify the coronal field modeling based on photospheric observations. If the flux surfaces and the current surfaces do not overlap, the current can simply leak through flux surfaces, and the values of $\alpha$ observed in the photosphere would be little relevant to the values in the coronal base. In this paper, we will derive the necessary and sufficient condition for magnetic flux-current surfaces to exist and will provide a theoretical criterion, by which one can tell either existence or inexistence of flux-current surfaces from photospheric observations.

By imposing some special assumptions, on the other hand, we may have

$$
\boldsymbol{B} \cdot \nabla \alpha=0,
$$

where

$$
\alpha \equiv \frac{\boldsymbol{J} \cdot \nabla \Phi}{\boldsymbol{B} \cdot \nabla \Phi}
$$

in a certain class of MHS equilibria as in Low (1991, 1993). Although this possibility looks very promising in constructing MHS equilibira or in force-free modeling based on photospheric data, the condition leading to it is found very restrictive. In this paper, we will illuminate the restrictions required for Eq. 12, particularly in relevance with the flux-current surfaces.

There have been quite a few theoretical works on MHS equilibrium (Low 1980, 1985, 1991, 2003, 2005; Tsinganos 1982; November 2004), among which a series of studies by Low (1985, 1991, 1993, 2005 among others) are considered to be so comprehensive and thorough that most theoretical works by others have been built upon them. Our study will not be an exception. It is already known that magnetic flux-current surfaces exist in 2.5D MHD configurations, in which all physical quantities including three components of vector fields depend on two coordinates only (e.g., Low 1993; Cheng $\&$ Choe 1998). As for the existence of magnetic fluxcurrent surfaces in general 3D MHS equilibria, no rigorous treatment has yet been presented. As will be shown later, the absence of a theory on this subject does not seem to be due to its difficulty, but rather to be attributed to unappreciation of its significance.

The followings are the tasks taken in our study as well as the contents of this paper. First, we will derive the necessary and sufficient condition for existence of magnetic flux-current surfaces in MHS equilibria in Section 2. Second in Section 3, we will examine the procedure leading to Eq. 12 by Low (1991) and generalize it to elaborate the conditions, under which Eq. 12 is valid. In Section 4, a discussion will be given on the force-free modeling of coronal magnetic fields, and a brief summary will conclude the paper.

\section{MAGNETIC FLUX-CURRENT SURFACES OF MHS EQUILIBRIA}

In this section, we will derive the necessary and sufficient condition for existence of magnetic flux-current surfaces in MHS equilibria. As with most other studies on MHS equilibrium, this work is also heavily indebted to Low (1991) and makes use of the materials presented in it, but rather in a critical manner. In order to help readers to have better understanding of both works, we will also use notations similar to those employed by Low (1991). 
Owing to the divergence-freeness of both $\boldsymbol{B}$ and $\boldsymbol{J}$, they can respectively be expressed in terms of a pair of Euler potentials (also called Clebsch variables) as

$$
\boldsymbol{B}=\nabla u \times \nabla v
$$

and

$$
\boldsymbol{J}=\nabla \mu \times \nabla \nu
$$

From the above relations, one can readily see that $\boldsymbol{B} \cdot \nabla u=0, \boldsymbol{B} \cdot \nabla v=0, \boldsymbol{J} \cdot \nabla \mu=0$, and $\boldsymbol{J} \cdot \nabla \nu=0$. A magnetic field line is the intersection of two surfaces $u=$ const. and $v=$ const. Thus, any iso-surfaces of $u$ or $v$ are magnetic flux surfaces. In the same way, any iso-surfaces of $\mu$ or $\nu$ are current surfaces. The existence of flux-current surfaces in an MHS equilibrium is equivalent to the existence of a scalar field $\chi$ such that

$$
\begin{aligned}
& \boldsymbol{B} \cdot \nabla \chi=0, \\
& \boldsymbol{J} \cdot \nabla \chi=0 .
\end{aligned}
$$

This $\chi$ can be called a flux-current surface label. If such a scalar field $\chi$ exists, $\chi$ can be one of the Euler potentials of $\boldsymbol{B}$ and at the same time one of the Euler potentials of $\boldsymbol{J}$. Then we can set $\chi=\mu$, and $u=\chi=\mu$ so that $\boldsymbol{B}=\nabla \mu \times \nabla v$ instead of Eq. 14 .

Substituting Eq. 14 into Eq. 1 and taking $u, v$ and $\Phi$ as coordinates, we have

$$
\begin{gathered}
\boldsymbol{J} \cdot \nabla u+\left.\frac{\partial p}{\partial v}\right|_{u, \Phi}=0 \\
\boldsymbol{J} \cdot \nabla v-\left.\frac{\partial p}{\partial u}\right|_{v, \Phi}=0 \\
\left.\frac{\partial p}{\partial \Phi}\right|_{u, v}+\rho=0 .
\end{gathered}
$$

These just reproduce Eqs. 5, 6 and 7 of Low (1991). On the other hand, substituting Eq. 15 into Eq. 1 and taking $\mu, \nu$ and $\Phi$ as coordinates, we have

$$
\begin{gathered}
\boldsymbol{B} \cdot \nabla \nu+\left.\frac{\partial p}{\partial \mu}\right|_{\nu, \Phi}=0, \\
\boldsymbol{B} \cdot \nabla \mu-\left.\frac{\partial p}{\partial \nu}\right|_{\mu, \Phi}=0, \\
\left.\frac{\partial p}{\partial \Phi}\right|_{\mu, \nu}+\rho=0 .
\end{gathered}
$$

These three are just the reproduction of Eqs. 11, 12 and 13 in Low (1991). Here it is to be noted that Eqs. 20 and 23 together imply

$$
\left.\frac{\partial p}{\partial \Phi}\right|_{\xi, \zeta}+\rho=0
$$

in any coordinate system $(\xi, \zeta, \Phi)$ with $\nabla \Phi \cdot(\nabla \xi \times \nabla \zeta) \neq$ 0 . Now we seek a necessary condition for existence of flux-current surfaces first. In order to do this, we suppose that flux-current surfaces exist in an MHS equilibrium and that $\mu$ be a flux-current surface label $(\boldsymbol{B} \cdot \nabla \mu=0$, and $\boldsymbol{J} \cdot \nabla \mu=0)$ and the common Euler potential of both $\boldsymbol{B}$ and $\boldsymbol{J}$ such that $\boldsymbol{B}=\nabla \mu \times \nabla v$, and $\boldsymbol{J}=\nabla \mu \times \nabla \nu$. On this supposition, $u$ in Eqs. $18-20$ should be replaced by $\mu$. Then, Eqs. 18 and 22 yield

$$
\left.\frac{\partial p}{\partial v}\right|_{\mu, \Phi}=0,\left.\quad \frac{\partial p}{\partial \nu}\right|_{\mu, \Phi}=0 .
$$

This implies that

$$
p=p(\mu, \Phi) .
$$

In other words, a necessary condition for existence of flux-current surfaces is that the plasma pressure $p$ should be a function of the gravitational potential and another scalar variable only. Is it the necessary and sufficient condition? In order to answer this question, we assume that the necessary condition is met, i.e., $p=p(\chi, \Phi)$. We do not know whether this $\chi$ satisfies Eqs. 16 and 17 or not. The existence of flux-current surfaces is equivalent to Eqs. 16 and 17 holding together, and this condition is again equivalent to

$$
\nabla p+\rho \nabla \Phi=\kappa \nabla \chi
$$

where $\kappa$ and $\chi$ are any scalar fields making this equation hold. Substituting this into Eq. 1 and taking a dot product with $\boldsymbol{B}$ and $\boldsymbol{J}$, respectively, will yield Eqs. 16 and 17. However, $\kappa$ and $\chi$ satisfying Eq. 27 do not always exist. If $p=p(\chi, \Phi)$, one can easily show that

$$
\nabla p+\rho \nabla \Phi=\left.\frac{\partial p}{\partial \chi}\right|_{\Phi} \nabla \chi
$$

using Eq. 24. Thus, this completes the proof that the necessary and sufficient condition for the existence of magnetic flux-current surfaces in an MHS equilibrium is that the plasma pressure should be a function of the gravitational potential and another scalar field only. This scalar field is constant along the magnetic field lines and along current lines and is a flux-current surface label.

The next question naturally raised is what this fluxcurrent surface label actually is. Now we name this flux-current surface label $\mu$. Thus, $p=p(\mu, \Phi)$. In each gravitational equipotential surface, $p=p(\mu)$, and iso-contours $p=$ const. and $\mu=$ const. overlap. We can choose one of gravitational equipotential surfaces as a reference surface. For this, the solar (stellar) surface can be taken, and field line footpoints are defined as the intersection of field lines and the reference surface. The gravitational potential in the reference surface is denoted by $\Phi_{0}$, and the pressure there by $p_{0}$. Since $p_{0}$ is a function of $\mu$ only, we can take for $\mu$ any function of $p_{0}$, i.e.,

$$
\mu=f\left(p_{0}\right), \quad p_{0}=f^{-1}(\mu),
$$


where $f$ is an arbitrary function of one variable. Of course, the simplest choice would be setting $\mu=p_{0}$. From now on, we will use this choice, which does not degrade the generality of our logic. If we interpret $p_{0}$ as the pressure at the footpoint of each field line, we can assign $p_{0}$ as a constant along each field line in the entire domain. However, such an assignment only does not necessarily guarantee the functional relation $p=p\left(p_{0}, \Phi\right)$ in the entire domain. In other words, the flux surface $\left(p_{0}=\right.$ const.) may not be the flux-current surface. This situation arises because the temperature profiles with height can be different for two field lines whose footpoint pressures are the same. Moving along two different field lines, we may have different amounts of heating, cooling and heat conduction at the same altitude. These are governed by local thermodynamics in each field line. Looking into the pressure profile along field lines can help illuminate this problem. We can easily obtain the pressure profile along a field line from the MHS equilibrium condition in the field direction (Eq. 20) and the equation of state (Eq. 5) (e.g., November 2004):

$$
p(u, v, \Phi)=p_{0}(u, v) \exp \left[-\int_{\Phi_{0}}^{\Phi} \frac{d \Phi}{R T(u, v, \Phi)}\right] .
$$

Here $T(u, v, \Phi)$ is a function governed by thermodynamics at each location. The existence of flux-current surfaces requires $p=p\left(p_{0}, \Phi\right)$, which again requires $T=T\left(p_{0}, \Phi\right)$, as can be seen in the above solution for $p$. Of course, there is no reason that two points located in different field lines at the same altitude have the same temperature just because they have the same footpoint pressure and temperature. However, that is what is mathematically required for flux-current surfaces to exist. Thus, the condition $p=p(\mu, \Phi)=p\left(p_{0}, \Phi\right)$ is regarded to be highly demanding.

Let us elaborate all the requirements connoted by the condition $p=p(\mu, \Phi)$ in a purely mathematical point of view. If $p=p(\mu, \Phi)$,

$$
\rho=\rho(\mu, \Phi),
$$

mandated by Eq. 24, because any partial derivative of $p(\mu, \Phi)$ is also a function $\mu$ and $\Phi$ only. From the equation of state (Eq. 5), we can see that

$$
T=T(\mu, \Phi) \text {. }
$$

Therefore, the iso-contours of $p, \rho$ and $T$ should all overlap in each gravitational equipotential surface as well as in the reference surface, and the iso-surfaces of $p, \rho$ and $T$ should all overlap in the entire domain. However, the above conditions are not all independent. The independent conditions are as follows. First, in one reference equipotential surface, two out of $p_{0}, \rho_{0}$ and $T_{0}$ iso-contours should overlap. Then, the third will automatically overlap with the other two by the equation of state (Eq. 5); hence $p_{0}=p_{0}(\mu), \rho_{0}=\rho_{0}(\mu)$, and $T_{0}=T_{0}(\mu)$. Second, in every equipotential surface, any two iso-contours out of three should overlap; then it follows that the third iso-contour overlaps with the other two. This condition is usually imposed by $T=T(\mu, \Phi)$, which is not automatically achieved by the MHS equilibrium condition, but to be imposed somehow. When all these requirements are fulfilled, we have $p=p(\mu, \Phi)$ and

$$
\boldsymbol{B} \cdot \nabla \mu=0
$$

and

$$
\boldsymbol{J} \cdot \nabla \mu=0 .
$$

Consequently flux-current surfaces $\mu=$ const. (or equivalently $p_{0}=$ const.) exist.

What then is the situation in which this demanding condition is satisfied so that flux-current surfaces exist? First, this condition is met if there is a geometrical symmetry and physical quantities also conform to this symmetry. For example, one can think of an axisymmetric sunspot structure, whether twisted or untwisetd, in which all physical quantities are assumed to be invariant in the azimuthal direction. In this case, a surface swept by one field line moving in the azimuthal direction is a flux-current surface. Not only the azimuthal symmetry, but any geometrical symmetry that allows us to ignore one coordinate dependence assures the existence of flux-current surfaces. If the coordinates are $(\xi, \eta, \zeta)$ and we can ignore the dependence on $\zeta$, the flux-current surfaces take a form $f(\xi, \eta)=$ const. In real $3 \mathrm{D}$ situations, an acceptable assumption that allows flux-current surfaces would be that $T=T(\Phi)$, i.e., the temperature depends on altitude only. This assumption is reasonably accommodated in a large scale modeling. If $T=T(\Phi)$, we only need to see to it that $\rho_{0}=f\left(p_{0}\right)$, i.e., the iso-contours of $p$ and $\rho$ overlap in the reference surface. If only this boundary condition is met, the condition $p=p\left(p_{0}, \Phi\right)$ is automatically satisfied in the entire domain in an MHS equilibrium. A purely isothermal case, where $T=$ const. everywhere, is merely a subset of cases with $T=T(\Phi)$. Oftentimes, we assume that the temperature is constant in each field line, but different in different field lines. This is a reasonable assumption for a coronal plasma in meso-scales because the field-aligned heat conduction is far more efficient than the field-perpendicular heat transport in the corona. This case is described by $T=T(u, v)$, where $u$ and $v$ are Euler potentials of $\boldsymbol{B}$. As can be seen in Eq. 30, this $T$-profile cannot make $p=p\left(p_{0}, \Phi\right)$. Unfortunately, flux surfaces and current surfaces do not overlap and there are generally no flux-current surfaces in this case.

\section{CONSTANTS IN FLUX-CURRENT SUR- FACES AND ALONG FIELD LINES}

\subsection{Constants in Magnetic Flux-current Sur- faces}

When magnetic flux-current surfaces exist in an MHS equilibrium, both magnetic flux and current 


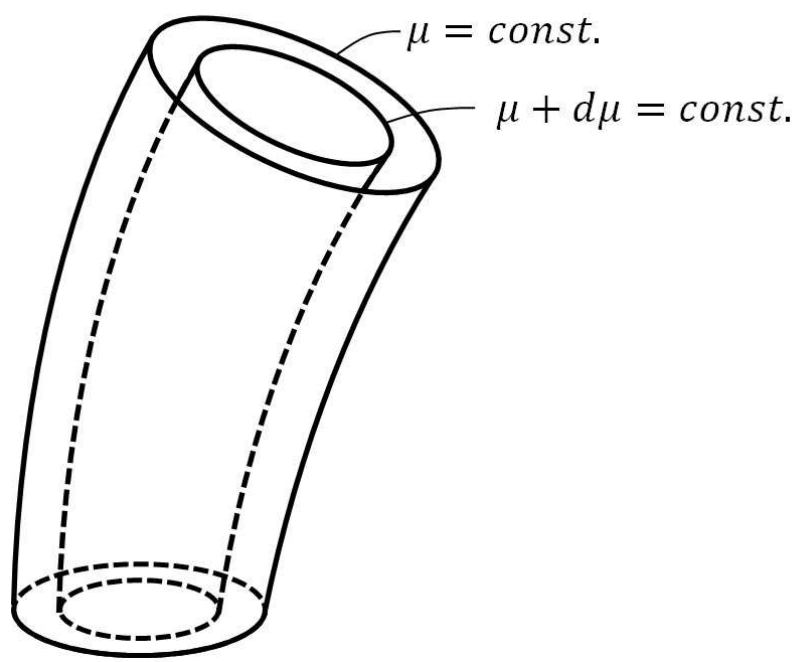

Fig. 1. - A sketch of magnetic flux-current surfaces labelled as $\mu$ and $\mu+d \mu$.

through a surface, whose boundary is a closed curve in a flux-current surface, are preserved. Consider a flux tube as shown in Fig. 1 with nested flux-current surfaces. Let $\mu$ be a flux-current surface label satisfying Eqs. 33 and 34. Suppose that a surface of an arbitrary shape intersects this flux tube. The current through an infinitesimal strip, which is a part of the surface cut by the flux-current surfaces $\mu$ and $\mu+d \mu$, is denoted by $d I$ and the magnetic flux through the same strip by $d \Psi$. Since all magnetic field lines and current lines in one flux-current surface stay in the same flux-current surface, $d I$ and $d \Psi$ are constant for any strip cutting the flux-current surfaces $\mu$ and $\mu+d \mu$. Thus,

$$
\tilde{\alpha}(\mu) \equiv \frac{d I}{d \mu} / \frac{d \Psi}{d \mu}
$$

is a preserved quantity along the flux tube. Also, the total current $I(\mu)$ and the total magnetic flux $\Psi(\mu)$ through a surface, which cuts the flux tube and is bounded by the flux-current surface $\mu$, are preserved along the flux tube, and so is their ratio

$$
\bar{\alpha}(\mu) \equiv I(\mu) / \Psi(\mu) .
$$

If magnetic flux-current surfaces do not exist, the current flowing between two current surfaces and the magnetic flux between two flux surfaces are preserved separately, but their ratio is not preserved along the flux tube because flux surfaces and current surfaces do not overlap.

\subsection{Generalization and Re-examination of the Formulation by Low (1991)}

Our analysis above indicates that there is no constant along each field line in general MHS equilibria even if flux-current surfaces exist. However, Low
(1991) showed that $\boldsymbol{B} \cdot \nabla \alpha=0$ (Eq. 12), where $\alpha=(\boldsymbol{J} \cdot \nabla \Phi) /(\boldsymbol{B} \cdot \nabla \Phi)$ (Eq. 13) under certain conditions. In this paper, we will examine the procedure leading to Eq. 12 and analyze the conditions for it. Low (1991) started with the following formulation, which can be applied to a certain class of twisted magnetic fields in MHD equilibrium.

$$
\boldsymbol{J}=\alpha \boldsymbol{B}+\nabla \mu \times \nabla \Phi .
$$

This equation can be rewritten with its content intact as follows.

$$
\boldsymbol{J}=\alpha \boldsymbol{B}+\boldsymbol{J}^{\prime},
$$

where

$$
\boldsymbol{J}^{\prime}=\nabla \mu \times \nabla \Phi .
$$

Since $\boldsymbol{J}$ is divided into $\alpha \boldsymbol{B}$ and $\boldsymbol{J}^{\prime}$ and $\boldsymbol{J}$ and $\boldsymbol{J}^{\prime}$ are divergence-free, $\alpha \boldsymbol{B}$ is also divergence-free, which results in Eq. 12. Low (1993) called the current system of an MHS equilibrium described by Eq. 38 as an "uncoupled" current system. If the current density field is divided into two terms, which are separately not divergence-free, it is called a "coupled" system. We will now make a little generalization of Eq. 38. In Eq. 38, $\boldsymbol{J}$ is expressed as a vector sum of $\alpha \boldsymbol{B}$ and $\boldsymbol{J}^{\prime}$. In general, a vector can be expressed as a linear combination of two non-parallel vector in a certain plane containing the three vectors. Thus, the formulation of Eq. 38 is based on the premises (i) that $\boldsymbol{J}, \boldsymbol{B}$ and $\boldsymbol{J}^{\prime}$ are locally coplanar and (ii) that $\boldsymbol{B}$ and $\boldsymbol{J}^{\prime}$ are non-parallel. The premise (ii) is acceptable because we are dealing with $\boldsymbol{B}$ and $\boldsymbol{J}$ not located in an equipotential surface. The premise (i) will soon be examined, but for the time being let us accept it. If the premises are accepted, $\boldsymbol{J}$ can be expressed as a linear combination of $\boldsymbol{B}$ and $\boldsymbol{J}^{\prime}$ as follows:

$$
\boldsymbol{J}=\alpha \boldsymbol{B}+\beta \boldsymbol{J}^{\prime} .
$$

The difference of Eq. 40 from 38 is the presence of the second coefficient (scalar field) $\beta$. With Eq. 38, one can express only a limited class of $\boldsymbol{J}$.

Now we further expand generality by re-defining $\boldsymbol{J}^{\prime}$ as the following:

$$
\boldsymbol{J}^{\prime}=\nabla \lambda \times \nabla \Phi
$$

where $\lambda$ is tentatively assumed to be an arbitrary scalar field. If we take a cross product of Eq. 1 with $\nabla \Phi$, we obtain Eq. 7 of Low (1993):

$$
\boldsymbol{J}=-\frac{1}{\boldsymbol{B} \cdot \nabla \Phi} \nabla p \times \nabla \Phi+\alpha \boldsymbol{B},
$$

which is exactly of the form of Eq. 40 with $\beta=$ $-(\boldsymbol{B} \cdot \nabla \Phi)^{-1}$ and $\lambda=p$ in Eq. 41. For the first term in the righthand side of this equation to be divergencefree, it is necessary that $B_{\Phi}=B_{\Phi}(p, \Phi)$, in which $B_{\Phi} \equiv \boldsymbol{B} \cdot \nabla \Phi$, as discussed in Low (1993). This condition means that $p=p\left(B_{\Phi}, \Phi\right)$, which is nothing but the necessary and sufficient condition for fluxcurrent surfaces to exist, as shown in Section 2. Thus, 
$B_{\Phi}=B_{\Phi}\left(p_{0}, \Phi\right)$. The existence of a current-flux surface is thus a necessary condition for the two current terms in Eq. 40 to be separately divergence-free (uncoupled), but is not a sufficient condition because the iso-surfaces of $B_{\Phi}$ must additionally overlap with the iso-surfaces of $p_{0}$ for uncoupled currents.

It should be noted that $\lambda$ in Eq. 41 does not need to be $p$. Now we will deal with general expressions with $\lambda \neq p$. We now substitute Eq. 40 with Eq. 41 into Eq. 1. Employing a coordinate system $(\lambda, \varpi, \Phi)$, where $\varpi$ is an arbitrary scalar field satisfying $\nabla \lambda \cdot(\nabla \varpi \times \nabla \Phi) \neq 0$ everywhere, and using Eq. 24, we have

$$
\boldsymbol{B} \cdot \nabla \lambda=0,
$$

which implies that $\lambda$ can be an Euler potential of $\boldsymbol{B}$,

$$
\left.\frac{\partial p}{\partial \varpi}\right|_{\lambda, \Phi}=0
$$

which means $p=p(\lambda, \Phi)$ in the formulation of Eq. 40, and

$$
\beta \boldsymbol{B} \cdot \nabla \Phi=-\left.\frac{\partial p}{\partial \lambda}\right|_{\Phi} .
$$

From Eq. 40, we have

$$
\boldsymbol{B}=\frac{1}{\alpha} \boldsymbol{B}-\frac{\beta}{\alpha} \boldsymbol{J}^{\prime} .
$$

Substituting this into Eq. 1 with Eq. 41 and using Eq. 24, we have

$$
\begin{gathered}
\boldsymbol{J} \cdot \nabla \lambda=0, \\
\frac{\beta}{\alpha} \boldsymbol{J} \cdot \nabla \Phi=-\left.\frac{\partial p}{\partial \lambda}\right|_{\Phi} .
\end{gathered}
$$

Eq. 47 implies that $\lambda$ can be an Euler potential of $\boldsymbol{J}$. This result with Eq. 43 tells that $\lambda$ is a flux-current surface label. Thus, the formulation given by Eq. 40 is possible only if magnetic flux-current surfaces exist. Since Eq. 37 employed in Low (1991) is just a special case of Eq. 40, that also presupposes the existence of flux-current surfaces. Since $\lambda$ is found to be a fluxcurrent surface label, we will from now on set $\lambda=\mu$ to recover our earlier notation. Also, we will introduce the following notations as in Low (1993):

$$
\begin{gathered}
B_{\Phi}=\boldsymbol{B} \cdot \nabla \Phi=-\boldsymbol{B} \cdot \boldsymbol{g}=B_{n} g, \\
J_{\Phi}=\boldsymbol{J} \cdot \nabla \Phi=-\boldsymbol{J} \cdot \boldsymbol{g}=J_{n} g,
\end{gathered}
$$

where $B_{n}$ and $J_{n}$, respectively, are upward normal components of $\boldsymbol{B}$ and $\boldsymbol{J}$ to the equipotential surface and $g=|\boldsymbol{g}|$.

Eqs. 45 and 48 are identical by the definition of $\alpha$ (Eq. 13). By setting $\lambda=\mu$, we rewrite it as

$$
\beta B_{\Phi}=-\left.\frac{\partial p}{\partial \mu}\right|_{\Phi} .
$$

Note that the righthand side is a function of $\mu$ and $\Phi$ only due to the existence condition of flux-current surfaces $p=p(\mu, \Phi)$. Hence, the lefthand side $\beta B_{\Phi}$ should be a function of $\mu$ and $\Phi$, but in general, $\beta$ and $B_{\Phi}$ are individually functions of three variables. Thus, neither terms in the righthand side of Eq. 40 is divergence-free in general although flux-current surfaces exist. In the case $\beta=1$ as in Low (1991), $B_{\Phi}$ is a function of $\mu$ and $\Phi$ only as discussed in Low (1993).

Taking divergence of Eq. 40, we have

$$
\boldsymbol{B} \cdot \nabla \alpha=-\boldsymbol{J}^{\prime} \cdot \nabla \beta .
$$

There is no reason that the righthand side of this equation is zero, and neither is the lefthand side. If $\beta=1$, we obtain $\boldsymbol{B} \cdot \nabla \alpha=0$ as in Low (1991).

\subsection{Imposition of Special Constraints}

We will approach the case by Low (1991) by gradually reducing generality, in other words, by increasing the number of constraints. One can see that the righthand side of Eq. 52 is zero when $\beta$ is a function of $\mu$ and $\Phi$ only because $\boldsymbol{J}^{\prime}=\nabla \mu \times \nabla \Phi$. Then, it follows that $\boldsymbol{B} \cdot \nabla \alpha=0$. Although the case $\beta=\beta(\mu, \Phi)$ is not very general, we will look into this special case. If we set

$$
\boldsymbol{J}^{\prime \prime} \equiv \beta \boldsymbol{J}^{\prime}=\beta \nabla \mu \times \nabla \Phi,
$$

and if $\beta=\beta(\mu, \Phi), \mu$ and $\Phi$ are so-called unmatched Euler potentials of $\boldsymbol{J}^{\prime \prime}$ and $\nabla \cdot \boldsymbol{J}^{\prime \prime}=0$. Thus, the constraint $\beta=\beta(\mu, \Phi)$ makes the two terms in the current density uncoupled (separately divergence-free). An unmatched Euler potential description can be converted to a regular Euler potential description by an appropriate transform of variables (Stern 1976). Particularly, if $\beta$ is a function of $\mu$ only, we can write

$$
\boldsymbol{J}^{\prime \prime}=\beta(\mu) \nabla \mu \times \nabla \Phi=\nabla F(\mu) \times \nabla \Phi,
$$

where $d F / d \mu=\beta(\mu)$. This recovers the formulation by Low (1991). If $\beta=\beta(\mu, \Phi)$, we see from Eq. 51 that $B_{\Phi}$ is also a function of $\mu$ and $\Phi$. This means that the isocontour of $\mu$ (or $p_{0}$ ) should overlap with the iso-contour of $B_{\Phi}$ in each gravitational equipotential surface. The equation $\boldsymbol{B} \cdot \nabla \alpha=0$ implies that $\alpha$ is constant along each field line. Since $\boldsymbol{B}=\nabla \mu \times \nabla v, \alpha=\alpha(\mu, v)$ and $\alpha$ does not explicitly depend on $\Phi$. Rewriting Eq. 48 as

$$
J_{\Phi}=-\left[\frac{\alpha(\mu, v)}{\beta(\mu, \Phi)}\right] \frac{\partial p(\mu, \Phi)}{\partial \mu}
$$

tells that $J_{\Phi}$ is not a function of $\mu$ and $\Phi$ only, but also of $v$. This is quite natural because there is no reason for the iso-contours of $B_{\Phi}$ and $J_{\Phi}$ to overlap in an equipotential surface even if $\beta=\beta(\mu, \Phi)$.

In Eq. 55, we can see that the iso-contours of $B_{\Phi}$ and $J_{\Phi}$ in every equipotential surface overlap if $\alpha$ is a function of $\mu$ only. In this case, $\boldsymbol{J} \cdot \nabla \alpha=0$, i.e., $\alpha$ is constant also along the current lines as well as along 
the magnetic field lines, and the iso-contours of $B_{\Phi}, J_{\Phi}$ and $\alpha$ all overlap in every equipotential surface if they overlap in the reference equipotential surface. Thus, an iso-surface of $\alpha$ is a flux-current surface overlapping with an iso-surface of $\mu$ (or $\left.p_{0}\right)$.

\section{DISCUSSION AND SUMMARY}

Only recently, there have been attempts to construct magnetohydrostatic equilibria with $3 \mathrm{D}$ currents to model the solar atmosphere and its magnetic field (Ruan et al. 2008; MacTaggart et al. 2013). For the practical purposes, however, the force-free field modeling (Wiegelmann \& Sakurai 2012; Wheatland \& Gilchrist 2013) prevails over the former, partly due to the lack of accurate observations of pressure in the photosphere and temperature in the entire atmosphere, and also due to the difficulty in the numerical MHS modeling. In force-free field modeling of coronal magnetic fields, $B_{n}$, the normal component of the magnetic field in the boundary, and $\alpha$ are the essential boundary conditions. These two quantities are mostly observed in the photosphere, whose state is far from force-free (Metcalf et al. 1995), and yet are used as boundary conditions in the coronal base. Both quantities are expected to vary from the photosphere to the coronal base. Among these two, $\alpha$ can be said to be the more pivotal quantity in determination of the overall field geometry (for example, how much the field is twisted). As shown in Section 3, the averaged value of $\alpha$ over a sectional strip between two flux surfaces (Eq. 35) or that over a cross-section of a flux tube (Eq. 36) is constant along the flux tube only when magnetic flux-current surfaces exist. The case, where $\alpha$ is constant along field lines, is a special subset of those cases as shown in Section 3.3. Therefore, the validity of force-free modeling of coronal magnetic fields based on photospheric data is largely dependent on whether magnetic fluxcurrent surfaces exist or not. We have presented the necessary and sufficient condition for the existence of magnetic-flux current surfaces in MHS equilibria. The following summary will give this condition again and tell how we can recognize existence and inexistence of magnetic flux-current surfaces.

The necessary and sufficient condition for an MHS equilibrium to have magnetic flux-current surfaces is that $p=p(\mu, \Phi)$, i.e., the pressure depends on the gravitational potential $\Phi$ (in most case a function of the altitude or the radial distance from the center of the star) and another scalar field $\mu$ only. This $\mu$ is identified to be a function of $p_{0}$ only; hence, we may simply set $\mu=p_{0}$. For the condition $p=p(\mu, \Phi)$ to hold in the whole domain, the condition $T=T(\mu, \Phi)$ must be fulfilled. Thus, we need to have an observational or theoretical ground before assuming $T=T(\mu, \Phi)$. For example, the system may have a geometrical symmetry or the temperature distribution may mostly be plane-parallel (or depending on the radial distance). Additionally, in the boundary (a reference equipotential surface), two of the iso-contours of $p_{0}, T_{0}$ and $\rho_{0}$ should overlap. Since this boundary condition is a necessary (not sufficient) condition for existence of magnetic flux-current surfaces, we can eliminate cases of inexistence by looking into the boundary data only.

We can decompose the current density as $\boldsymbol{J}=\alpha \boldsymbol{B}+$ $\beta \boldsymbol{J}^{\prime}$, where $\boldsymbol{J}^{\prime}=\nabla \mu \times \nabla \Phi$. When both $\alpha \boldsymbol{B}$ and $\beta \boldsymbol{J}^{\prime}$ are divergence-free, we call the current uncoupled, and otherwise coupled. When flux-current surfaces do not exist, we cannot have an uncoupled current system. When flux-current surfaces exist, we can have either coupled current systems or uncoupled current systems. When $\beta=\beta(\mu, \Phi)$, an uncoupled system arises and it follows that $B_{\Phi}=B_{\Phi}(\mu, \Phi)$ and $\boldsymbol{B} \cdot \nabla \alpha=0$. In a further restricted case $\alpha=\alpha(\mu), \boldsymbol{J} \cdot \nabla \alpha=0$. Thus, the iso-surface of $\alpha$ can be a flux-current surface only in this very special case. In this case, the iso-contours of $p_{0}, T_{0} \rho_{0}, B_{\Phi}, J_{\Phi}$, and $\alpha$ all overlap in the boundary. It is hard to imagine that such a situation arises except when a geometrical symmetry exists or when $T=T(\Phi)$ and $\alpha=$ const. everywhere.

\section{ACKNOWLEDGMENTS}

This work was supported by Basic Science Research Program through the National Research Foundation of Korea (NRF) funded by the Ministry of Education (NRF-2013R1A1A2058937). The authors thank the anonymous referee for helpful comments in improving the paper. They thank Mr. Hongdal Jun for preparing a figure in the paper. G. S. C. expresses thanks to Kyung Hee University for granting him a sabbatical in 2012-2013 school year, during which part of this research was performed. He also extends his sincere gratitude to Profs. S. K. Solanki and J. Buechner of Max Planck Institute for Solar System Research for the support and hospitality provided to him during his sabbatical stay there as well as for fruitful scientific discussions.

\section{REFERENCES}

Aly, J. J. 1989, On the Reconstruction of the Nonlinear Force-Free Coronal Magnetic Field from Boundary Data, Sol. Phys., 120, 19

Amari, T., Aly, J. J., Luciani, J. F., Boulmezaoud, T. Z., \& Mikic, Z. 1997, Reconstructing the Solar Coronal Magnetic Field as a Force-Free Magnetic Field, Sol. Phys., 174, 129

Cheng, C. Z., \& Choe, G. S. 1998, Current Sheets and Prominence Formation in the Solar Atmosphere, ApJ, 505, 376

Choe, G. S., \& Cheng, C. Z. 2000, A Model of Solar Flares and Their Homologous Behavior, ApJ, 541, 449

D'haeseleer, W. D., Hitchon, W. N. G., Callen, J. D., \& Shohet, J. L. 1991, Flux Coordinates and Magnetic Field Structure (Berlin: Springer-Verlag), 64 
Grad, H., \& Rubin, H. 1958, Hydromagnetic Equilibria and Force-Free Fields, in Proc. 2nd Int. Conf. on Peaceful Uses of Atomic Energy, Vol. 31 (Geneva: United Nations), 190

Low, B. C. 1980, On Magnetostatic Equilibrium in a Stratified Atmosphere, Sol. Phys., 65, 147

Low, B. C. 1985, Three-Dimensional Structures of Magnetostatic Atmospheres. I. Theory, ApJ, 293, 31

Low, B. C. 1991, Three-Dimensional Structures of Magnetostatic Atmospheres. III. A General Formulation, ApJ, 370, 427

Low, B. C. 1993, Three-Dimensional Structures of Magnetostatic Atmospheres. V. Coupled Electric Current Systems, ApJ, 408, 689

Low, B. C. 2005, Three-Dimensional Structures of Magnetostatic Atmospheres. VII. Magnetic Flux Surfaces and Boundary Conditions, ApJ, 625, 451

MacTaggart, D., Elsheikh, A., McLaughlin, J. A., \& Simitev, R. D. 2013, Non-Symmetric Magnetohydrostatic Equilibria: A Multigrid Approach, A\&A, 556, A40

McClymont, A. N., Jiao, L., \& Mikic, Z. 1997, Problems and Progress in Computing Three-Dimensional Coronal Active Region Magnetic Fields from Boundary Data, Sol. Phys., 174, 191

Metcalf, T. R., Jiao, L., McClymont, A. N., Canfield, R. C., \& Uitenbroek, H. 1995, Is the Solar Chromospheric Magnetic Field Force-Free?, ApJ, 439, 474

November, L. J. 2004, Magnetohydrostatic (MHS) Atmospheres, A\&A, 417, 333

Parker, E. N. 1979, Cosmical Magnetic Fields (Oxford: Oxford University Press), 63

Ruan, P., Wiegelmann, T., Inhester, B., Neukirch, T., Solanki, S. K., \& Feng, L. 2008, A First Step in Reconstructing the Solar Corona Self-Consistently with a Magnetohydrostatic Model during Solar Activity Minimum, A\&A, 481, 827

Sakurai, T. 1989, Computational Modeling of Magnetic Fields in Solar Active Regions, Space Sci. Rev., 51, 11

Schrijver, C. J., De Rosa, M. L., Metcalf, T. R., Liu, Y., McTiernan, J., Régnier, S., Valori, G., Wheatland, M. S., \& Wiegelmann, T. 2006, Nonlinear ForceFree Modeling of Coronal Magnetic Fields Part I: A Quantitative Comparison of Methods, Sol. Phys., 235,161

Stern, D. P. 1976, Representation of Magnetic Fields in Space, Rev. Geophys. Space Phys., 14, 199

Tsinganos, K. C. 1982, Magnetohydrodynamic Equilibrium. II. General Integrals of the Equations with One Ignorable Coordinate, ApJ, 252, 775

Wheatland, M. S., \& Gilchrist, S. A. 2013, Journal of Physics Conference Series, 440, 012037

Wiegelmann, T., \& Sakurai, T. 2012, Solar Force-Free Magnetic Fields, Living Reviews in Solar Physics, 9, 5 